\title{
THE UNIFICATION OF QUASARS AND RADIO GALAXIES
}

\author{
SPERELLO DI SEREGO ALIGHIERI \\ Osservatorio Astrofisico di Arcetri \\ Largo E. Fermi, 5 - 50125 Firenze - Italy
}

\section{Introduction}

The evidence for misdirected quasars in the nuclei of powerful radio galaxies (PRG) is now solid: 1. strong perpendicular polarization of the UV continuum, produced by the scattering of light from the hidden nucleus, has been observed in 10 (out of 11) PRG with $z>0.7$ (Cimatti et al. 1993); 2. polarized broad MgII2800 has been seen in 6 PRG (di Serego Alighieri et al. 1994, Antonucci et al. 1994), showing that the hidden nucleus is indeed a quasar. These findings are receiving authoritative confirmation from observations with the Keck telescope (Cimatti, Cohen and van Breugel in these Proceedings). Here I would like to concentrate on recent developments of our understanding of the unification between quasars and PRG.

\section{A dust scattering model for powerful radio galaxies}

With Alessandro Manzini we have developed a model to reproduce the spectral energy distribution (SED) and the polarization of the light from the hidden quasar which is scattered in our direction by dust in the ISM of the PRG (Manzini and di Serego Alighieri 1995). This model uses Mie single scattering, the MRN dust size distribution and the geometry suggested by the Unified Model, and deals consistently with extinction and scattering by the same dust. The main results are: 1 . the dust scattering efficiency can be either blue, red or flat, depending on the amount of dust; therefore a flat scattered light does not necessarily imply electron scattering. 2. The $2175 \AA$ feature, which appears in emission in the MRN albedo, but is not generally observed in the SED of PRG at high redshift, can be eliminated including a proper amount of extinction. 3. The polarization resulting from the model is between 5 and $50 \%$, not uncomfortably higher than the observed values, and varies with the mean scattering angle. 
This model, together with improved stellar population synthesis models (D. Villani in these Proceedings) and a model of the nebular continuum (Dickson et al. 1995), have been used to fit the SED and the polarization observed in a number of PRG, giving constraints on the luminosity of the hidden quasar, on the dust content, and on the stellar population ages in distant PRG.

\section{3. [OIII] anisotropies in powerful radio galaxies}

Jackson and Browne (1990) have shown that the [OIII] luminosity of quasars is larger than that of PRG for a sample of radio sources at intermediate redshifts. This result has been regarded as a failure of the Unified Model, since the [OIII] line was believed to be radiated isotropically and then it should be observed independently of orientation. However high ionization lines with a large critical density could be emitted close enough to the nucleus to be partially within the obscuring "torus" and therefore escape anisotropically. We have made spectropolarimetric observations of 6 PRG and of 3 quasars to detect possible anisotropies in the [OIII] emission of PRG. We have preliminarily detected polarized [OIII] in 5 PRG, indicating anisotropic emission, while [OIII] is not polarized in the 3 quasars observed (di Serego Alighieri et al. in preparation).

\section{Outlook}

These recent improvements in our understanding of the active components of PRG are leading to a refinement of the Unified Model of the most powerful AGN, and give us confidence that also stellar populations can be studied in these objects at cosmological distances. Further improvements can be expected in the following areas: studies of how the importance of anisotropic emission in AGN changes with redshift and radio power, estimates of the dilution in the UV polarization in PRG from detailed spectropolarimetry of broad emission lines, studies of the IR polarization and of the $2175 \AA$ feature as a dust diagnostic, searches for stellar and interstellar absorptions, and the development of inhomogeneous dust scattering models.

\section{References}

Antonucci, R., Hurt, T. \& Kinney, A., 1994, Nature, $371,313$.

Cimatti, A., di Serego Alighieri, S., Fosbury, R.A.E., Salvati, M. \& Taylor, D., 1993, M.N.R.A.S., $264,421$.

di Serego Alighieri, S., Cimatti, A. \& Fosbury, R.A.E., 1994, ApJ, 431, 123.

Dickson, R., Tadhunter, C., Shaw, M., Clark, N. \& Morganti, R., 1995, M.N.R.A.S., 273, L29.

Jackson, N. \& Browne, I.W.A., 1990, Nature, 342, 43.

Manzini, A. \& di Serego Alighieri, S., 1995, $A \& A$, submitted. 